\title{
Retraction Note: Addiction of drugs like Cocaine and Opium - A Review
}

Sonia Bidhan and Anu Radha Pathania

Published online: 7 October 2021

Retracted article: E3S Web of Conferences 309, 01151 (2021)

https://doi.org/10.1051/e3sconf/202130901151

This paper has been formally retracted on ethical grounds because the article contains instances of plagiarism, without proper citation. The authors are responsible for this mistake and apologise for it. Request approved by the proceedings Editor and the Publisher on December 10, 2021. 